\title{
Carboxyl-terminal modulator protein induces apoptosis by regulating mitochondrial function in lung cancer cells
}

\author{
SOON-KYUNG HWANG ${ }^{1,2^{*}}$, ARASH MINAI-TEHRANI ${ }^{1 *}$, KYEONG-NAM YU $^{1}$, SEUNG-HEE CHANG ${ }^{1}$, \\ JI-EUN KIM ${ }^{1,3}$, KEE-HO LEE ${ }^{5}$, JONGSUN PARK ${ }^{6}$, GEORGE R. BECK Jr ${ }^{7}$ and MYUNG-HAING CHO ${ }^{1,3,4}$ \\ ${ }^{1}$ Laboratory of Toxicology, College of Veterinary Medicine Seoul National University, Seoul 151-742, Republic of Korea; \\ ${ }^{2}$ Gene Regulation Section, Laboratory of Cancer Prevention, Center for Cancer Research, National Cancer Institute, Frederick, \\ MD 21702, USA; ${ }^{3}$ Department of Nano Fusion Technology, Graduate School of Convergence Science and Technology, \\ ${ }^{4}$ Graduate Group of Tumor Biology, Seoul National University, Seoul 151-742; ${ }^{5}$ Laboratory of Molecular Oncology, \\ Division of Radiation Cancer Research, Korea Institute of Radiological and Medical Sciences, Seoul 139-240; \\ ${ }^{6}$ Department of Pharmacology, Daejeon Regional Cancer Center, Cancer Research Institute, Research Institute \\ for Medical Sciences, College of Medicine, Chungnam National University, Daejeon 301-131, Republic of Korea; \\ ${ }^{7}$ Division of Endocrinology, Metabolism and Lipids, Emory University School of Medicine, Atlanta, GA 30322, USA
}

Received September 24, 2011; Accepted November 1, 2011

DOI: $10.3892 /$ ijo.2011.1319

\begin{abstract}
Serine/threonine protein kinase B (PKB/Akt) is involved in cell survival and growth. Carboxyl-terminal modulator protein (CTMP), a novel Akt binding partner, prevents Akt activation at the plasma membrane in response to various stimuli, and thus possesses a tumor suppressor-like function. In a previous study, we have demonstrated that CTMP inhibits tumor progression by facilitating apoptosis in a mouse lung cancer model. However, the precise mechanism of CTMPinduced apoptosis remains to be elucidated. The present study was performed to examine the role of CTMP in mitochondrialmediated apoptosis and regulation of mitochondrial function in human lung carcinoma cells. Our results showed that CTMP altered mitochondrial morphology and caused the release of cytochrome $c$ by inhibiting OPA1 expression. Additionally, CTMP facilitated mitochondrial-mediated apoptosis by inhibiting heat-shock protein 27 and preventing cytochrome $c$ interaction with Apaf-1. Our data suggest that CTMP may therefore play a critical role in mitochondrial-mediated apoptosis in lung cancer cells.
\end{abstract}

\section{Introduction}

Lung cancer is the leading cause of cancer-related deaths in the world with over one million cases diagnosed every year. Multiple

Correspondence to: Dr Myung-Haing Cho, Laboratory of Toxicology, College of Veterinary Medicine, Seoul National University, Seoul 151-742, Republic of Korea

E-mail mchotox@snu.ac.kr

*Contributed equally

Key words: CTMP, apoptosis, mitochondria, lung cancer cells options for lung cancer treatment have been described including surgery, chemotherapy, and radiation, however, the therapeutic effects are typically transient and mostly absent in cases of advanced disease (1). Therefore, a more rational approach to lung cancer therapy is essential.

Mitochondria do not only supply ATP but also participate in regulatory and signaling events, respond to multiple physiological inputs and genetic stresses, thus, regulate cell proliferation and death (2). Recently, many studies have demonstrated that mitochondria form dynamic tubular networks via controlling fusion and fission in response to diverse external stimuli. Moreover, the proteins involved in mitochondria fission and fusion actively participate in apoptosis (3).

The serine-theronine kinase Akt (protein kinase B) is an important regulator of cell survival (4). Activated Aktl is localized to various subcellular compartments, including golgi, endoplasmic reticulum, mitochondria, and nucleus where it phosphorylates substrates and interacts with other molecules (5). Constitutive Akt activation greatly contributes to aberrant cell cycle regulation, a hallmark of many cancers, resulting in uncontrolled cell proliferation and suppression of apoptotic pathway. In fact, Akt can modulate apoptosis directly or indirectly (6). The direct effects are related to phosphorylation or interactions with factors involved in cell death, whereas the indirect regulation of apoptosis is mediated through transcriptional responses to apoptotic stimuli. In both cases, Akt is at the crossroads of several mitochondria-mediated cell death pathways, therefore, it can be an important target for cancer therapy.

Carboxyl-terminal modulator protein (CTMP) is an Akt binding partner. CTMP overexpression inactivates Akt in $v$-Akttransformed cells transplanted in mice (7), cultured cell (8) and K-ras lung cancer model mice (9). So far, the biological mechanism through which CTMP regulates mitochondrial functions and apoptosis is still poorly understood. Therefore, the current study was performed to investigate the detailed role of CTMP in mitochondria functions related to apoptosis. Our results 
demonstrated that CTMP altered mitochondria morphology by inhibiting OPA1, leading to cytochrome $c$ release from mitochondria. Finally, CTMP facilitated apoptosis by inhibiting the anti-apoptotic heat-shock protein 27 (Hsp27). We report that CTMP is involved in mitochondria-mediated apoptotic processes and the regulation of mitochondria dynamics. Our results support the hypothesis that CTMP overexpression may promote apoptosis in lung cancer cells.

\section{Materials and methods}

Antibodies and reagents. Monoclonal antibody against CTMP was generated using a previously described general method (14). Anti-OPA1 (optic atrophy 1) antibody was purchased from Novus Biological (Littleton, CO, USA). Anti-COX IV, anti-Hsp60, anti-Apaf-1, anti-MFN1 (mitofusin1), anti-MFN2 (mitofusin2), anti-DRP1 (dynamin-related protein 1), anti-active caspase-3 and anti-AIF (apoptosis-inducing factor) antibodies were purchased from Abcam (Cambridge, MA, USA). Anticytochrome $c$ and anti-Hsp27 antibodies were from Santa Cruz Biotechnology (Santa Cruz, CA, USA). Anti-cleaved PARP [poly ADP (adenosine diphosphate)-ribose polymerase] antibody was obtained from Cell Signaling (Danvers, MA, USA). HRP conjugated anti-mouse and anti-rabbit IgG antibodies were from Zymed (San Francisco, CA, USA).

Cell culture and lentivirus infection. Human non-small cell lung cancer A549 cells were purchased from the American Tissue Type Culture Collection (ATCC, Manassas, VA, USA). The cells were maintained in RPMI-1640 (Invitrogen, Carlsbad, CA, USA) with $10 \%$ heat-inactivated fetal calf serum (FCS), 100,000 U/1 penicillin, and $100 \mathrm{mg} / \mathrm{l}$ streptomycin (Invitrogen) at $37^{\circ} \mathrm{C}$ in $5 \% \mathrm{CO}_{2}$ incubator. Stable cell lines expressing pDsRed-Mito were isolated after selecting for 3 weeks with G418 (400 $\mu \mathrm{g} / \mathrm{ml}$, Sigma-Aldrich, St. Louis, MO, USA). Lentivirus was prepared using a lentiviral expression kit (Invitrogen). A549 cells were infected with appropriate concentration (10 ng/ml) of lentivirus-CTMP (CTMP) and lentivirus expression vector (VEC) for $24 \mathrm{~h}$. The medium was then changed and the cells were further incubated for another $48 \mathrm{~h}$ before harvesting.

Detection of cell apoptosis. Cells were cultured in a T-75 flask and infected with lentivirus-CTMP and lentivirus expression vector for $24 \mathrm{~h}$. The cells were then trypsinized, collected, and washed. Apoptosis was evaluated by terminal deoxynucleotidyl transferase-mediated dUTP-nick end-labeling (TUNEL) using a APO-BrdU TM TUNEL assay kit (Invitrogen) according to the manufacturer's instructions and analyzed by an FACSCalibur (BD Bioscience, Franklin Lakes, NJ, USA).

Small interference RNA (siRNA) transfection. A549 cells were grown to $70 \%$ confluence, $10 \mathrm{nM}$ of chemically synthesized siRNA targeting CTMP or non-targeting 20-25 nt siRNA (control siRNA) were transfected using Santa Cruz transfection reagent according to the manufacturer's instructions (Santa Cruz Biotechnology). Briefly, transfection reagent was incubated with serum-free media for $10 \mathrm{~min}$. Subsequently, a mixture of each respective siRNAs was added. After incubation for $15 \mathrm{~min}$ at room temperature, the mixture was added to each well. After incubating for $6 \mathrm{~h}$, the cells were washed and fresh culture media were added. The cells were then harvested after 12,24 , and $48 \mathrm{~h}$.

Transmission electron microscopy (TEM). A549 cells were fixed with a solution of $2.5 \%$ glutaraldehyde with $1 \%$ osmium tetroxide $\left(\mathrm{OsO}_{4}\right)$ buffer for $2 \mathrm{~h}$ at $4^{\circ} \mathrm{C}$ and dehydrated with ethanol at $4^{\circ} \mathrm{C}$. The cells were then infiltrated in a 1:1 mixture of propylene oxide and Epon, and finally embedded in Epon by polymerization at $70^{\circ} \mathrm{C}$ for $24 \mathrm{~h}$. Ultrathin sections $(40-70 \mathrm{~nm})$ were cut and mounted on pioloform-coated copper grids. Sections were stained with lead citrate and uranyl acetate, and viewed with a JEM 1010 transmission electron microscope (Jeol, Tokyo, Japan).

Confocal imaging analysis and indirect immnuofluorescence. Cells stably expressing pDsRed-Mito were grown in 2-well chamber slides until they were 50-70\% confluent, and then infected with VEC or lentivirus-CTMP. The cells were fixed with $4 \%$ paraformaldehyde at room temperature for $10 \mathrm{~min}$, mounted with DakoCytomation Faramount Aqueous Mounting solution (DakoCytomation, Carpinteria, CA, USA) and visualized using a Carl Zeiss confocal microscope (Carl Zeiss MicroImaging, Thronwood, NY, USA). For indirect immunofluorescence, A549 cells were fixed with 4\% paraformaldehyde and permeabilized with $1 \%$ Triton X-100 at room temperature for $15 \mathrm{~min}$. The cells were then incubated in blocking buffer containing 3\% bovine serum albumin (Sigma-Aldrich) in $1 \mathrm{X}$ TBS for $1 \mathrm{~h}$ at $37^{\circ} \mathrm{C}$. The cells were incubated overnight with primary antibodies diluted 200 -fold. Primary antibody binding was detected using a FITC-conjugated secondary antibody (BD Biosciences). After rinsing, coverslips were mounted in DakoCytomation Faramount Aqueous Mounting solution on glass slides and analyzed.

Isolation of mitochondria and cytosolic protein. Infected cells were washed with PBS and resuspended in mitochondrial fraction buffer (20 mM HEPES, pH 8.0; $10 \mathrm{mM} \mathrm{KCl,} 1.5 \mathrm{mM}$ $\mathrm{MgCl}_{2}, 1 \mathrm{mM}$ EDTA, $250 \mathrm{mM}$ sucrose, $1 \mathrm{mM}$ PMSF, $10 \mathrm{~g} / \mathrm{ml}$ leupeptin, $10 \mathrm{~g} / \mathrm{ml}$ aprotinin, and $0.2 \mathrm{mM}$ sodium orthovanadate) for $30 \mathrm{~min}$ on ice, and then homogenized. Unbroken cells and nuclei were pelleted by centrifugation at $1500 \mathrm{x} \mathrm{g}$ for $10 \mathrm{~min}$. The supernatant was centrifuged at $10,000 \mathrm{x}$ g for $30 \mathrm{~min}$ at $4^{\circ} \mathrm{C}$ and transferred to a new tube to obtain the post-mitochondrial fraction. Next, the supernatant was centrifuged at $100,000 \mathrm{x} g$ for $1 \mathrm{~h}$ at $4^{\circ} \mathrm{C}$ to recover the cytosolic fraction. The post-mitochondrial fraction pellet was washed with $500 \mu \mathrm{l}$ of mitochondrial fraction buffer and used to isolate the mitochondrial fraction. The supernatant and mitochondrial fractions were collected and total protein concentration was determined by Bradford assay. Equivalent amounts of protein were loaded, separated on SDS-polyacrylamide gels, and transferred to a nitrocellulose membrane. The membrane was incubated with mouse anti-cytochrome $c$ (Santa Cruz) and rabbit anti-HSP60 (Abcam), followed by incubation with HRP-conjugated secondary antibodies. Release of cytochrome $c$ from the mitochondria into the cytosol of A549 cells was measured with a human cytochrome $c$ ELISA kit (R\&D Systems, Minneapolis, MN, USA). Cytochrome $c$ was quantified using an ELISA reader (Bio-Rad, Hercules, CA, USA). 
Western blot analysis. Cells were placed on ice and incubated in lysis buffer containing $50 \mathrm{mM}$ Tris- $\mathrm{HCl}, \mathrm{pH} 7.5,1 \%$ (v/v) Nonidet P-40, $120 \mathrm{mM} \mathrm{NaCl}, 25 \mathrm{mM}$ sodium fluoride, $40 \mathrm{mM}$-glycerol phosphate, $0.1 \mathrm{mM}$ sodium orthovanadate, $1 \mathrm{mM}$ phenylmethylsulfonyl fluoride, $1 \mathrm{mM}$ benzamidine, and $2 \mu \mathrm{M}$ microcystin-LR). Lysates were centrifuged for $15 \mathrm{~min}$ at $12,000 \mathrm{x}$ g. Total proteins from the cell extracts were resolved with $12 \%$ SDS-PAGE and transferred to nitrocellulose membranes (Amersham Pharmacia, Pittsburgh, PA, USA). The membranes were blocked for $1 \mathrm{~h}$ in TTBS (Tris-buffered saline and Tween-20) containing 5\% skim milk. Immunoblotting was done by incubating overnight at $4^{\circ} \mathrm{C}$, and then with secondary HRP-conjugated antibodies for $3 \mathrm{~h}$ at room temperature or overnight at $4^{\circ} \mathrm{C}$. After the blots were washed, the bands were observed with a LAS-3000 luminescent image analyzer (Fujifilm, Tokyo, Japan) and quantified using the multi Gauge version 2.02 program (Fujifilm).

Mitochondrial membrane potential analysis. To analyze the $\Delta \Psi_{\mathrm{m}}$, A549 cells were cultured in a 2-well chamber slide and infected with lentivirus-CTMP and lentivirus for $24 \mathrm{~h}$. After $48 \mathrm{~h}$, cells were collected and washed with PBS, and then resuspended in $500 \mu \mathrm{l}$ PBS. The resuspened cells were labeled with $5 \mu \mathrm{g} / \mathrm{ml} \mathrm{JC}$-1 (5,5',6',6'-tetrachloro-1,1',3,3'-tetaethylbenzimidazolylcarbocyanine iodide, Molecular Probes) at $37^{\circ} \mathrm{C}$ in the dark for $30 \mathrm{~min}$. Labeled cells were washed once in PBS and analyzed with a FACSCalibur (BD Bioscience).

Quantification of Apaf-1 protein using flow cytometry. Cells expressing CTMP and control cells were collected. Cells were then washed in cold phosphate-buffered saline (PBS) before being incubated with rabbit anti-human Apaf-1 monoclonal antibody for $1 \mathrm{~h}$ at room temperature in the dark. After washing twice with PBS, FITC-conjugated anti-rabbit secondary antibody was added for $30 \mathrm{~min}$. After washing, the stained cells were analyzed by flow cytometry (BD Bioscience).

Active caspase 3 ELISA assay. Levels of active caspase-3 were quantitated using a human active-caspase-3 immunoassay kit according to the manufacturer's instructions (R\&D Systems). Briefly, caspase-3 was labeled in cells with biotin-ZVKD-fmk for $1 \mathrm{~h}$. The cells were then rinsed with PBS, and extraction buffer containing urea and protease inhibitors were added to each well. The cells were incubated overnight at $4^{\circ} \mathrm{C}$ and cell lysates were added to the active-caspase- 3 microplate, and the plate was incubated for $2 \mathrm{~h}$ at room temperature. Active caspase- 3 conjugate was then added and the plate was incubated for $1 \mathrm{~h}$. After substrate was added and the reaction was quenched according to the manufacturer's instructions, the plates were read at $450 \mathrm{~nm}$ with a wavelength correction at $540 \mathrm{~nm}$. A standard curve was constructed and the level of active-caspase- 3 in each sample was calculated.

Cleaved PARP ELISA assay. The cleaved PARP in the cell lysates of A549 cells was measured by a sandwich ELISA kit (Cell Signaling Technology, Danvers, MA, USA). The cleaved PARP molecules in the cells were captured into the wells by specific antibody. Captured cleaved PARP molecules were sandwiched by a monoclonal cleaved PARP antibody and detected by secondary antibody. TMB substrate was used to develop the color, plates were read at $450 \mathrm{~nm}$ in an ELISA plate reader (Bio-Rad).

Statistical analysis. Data are expressed as the mean \pm SE of at least three separate experiments performed in triplicate. Differences between groups were analyzed using Student's t-test (Graphpad Software, San Diego, CA, USA). A p $<0.05$ were considered significant, and a $\mathrm{p}<0.01$ was considered to be highly significant.

\section{Results}

Localization of CTMP in mitochondria. To evaluate the intracellular localization of CTMP, A549 cells were fractionated into total, cytosolic, and mitochondria fractions. As shown in Fig. 1A, CTMP was detected in the mitochondria fraction. Confocal microscopy analysis of A549 cells co-transfected with GFP-N-CTMP and pDsRed-Mito (mitochondria marker) confirmed that CTMP was co-localized to mitochondria with Red-Mito in A549 cells (Fig. 1B and C). To further determine the precise localization of CTMP in the mitochondrial structure, mitochondria were subfractionated and then incubated with $2 \mathrm{M} \mathrm{NaCl}$ for mitochondria outer-membrane and $100 \mathrm{mM} \mathrm{Na} \mathrm{CO}_{3}$ for inner-membrane space. Our data showed that CTMP was solublized in $\mathrm{Na}_{2} \mathrm{CO}_{3}$, indicating that CTMP is a mitochondrial inner membrane protein (Fig. 1D). Immunofluroscence analysis using anti-AIF (an inner-membrane marker) antibody clearly showed co-localization of CTMP and AIF protein (Fig. 1E).

Overexpression of CTMP sensitizes the cells to apoptosis induced by staurosporine and actinomycin D. To determine whether CTMP has an effect on cell death, A549 cells were infected with lentivirus-CTMP and apoptosis was induced using actinomycin D and staurosporine. CTMP overexpression increased the expression of cleaved PARP and active-caspase- 3 after apoptosis was induced as determined by Western blotting (Fig. 2A and B). Increased PARP and caspase-3 expressions were reduced by siRNA CTMP, such decreased CTMP level using siRNA was further confirmed by densitometric analysis (Fig. 2C).ELISA assay also clearly showed that CTMP increased the expression of active caspase-3 and cleaved PARP (Fig. 2D and E). Quantitative analysis also confirmed the significant increase of apoptotic-positive cells following CTMP treatment compared to the control (Fig. 2F).

CTMP alters OPA1 expression andmitochondrialmorphology. Mitochondrial fusion in mammalian cells is controlled by the GTPases Mfn1, Mfn2, and OPA1. It has been reported that decreases in mitochondrial fusion proteins induces apoptosis (10). To further evaluate the link between CTMP-induced apoptosis and these proteins, we investigated changes in the expression of MFN1, MFN2, and OPA1. We did not observe any significant changes in Mfn1 and Mfn2 expression while OPA1 protein was markedly reduced in CTMP-infected cells (Fig. 3A and B). Our results clearly showed a significant decrease in OPA1 expression after CCCP treatment while this was increased by CTMP-specific siRNA (Fig. 3B).

Downregulation of OPA1 not only induces spontaneous apoptosis and mitochondria fragmentation but also disrupts 
A

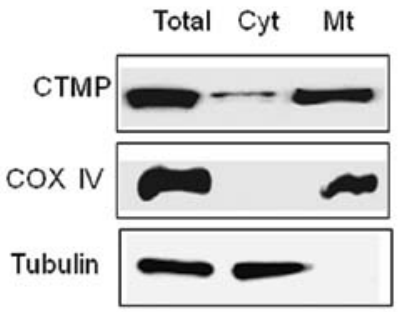

B
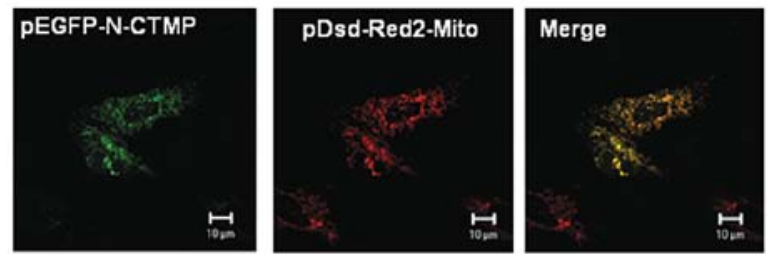

C
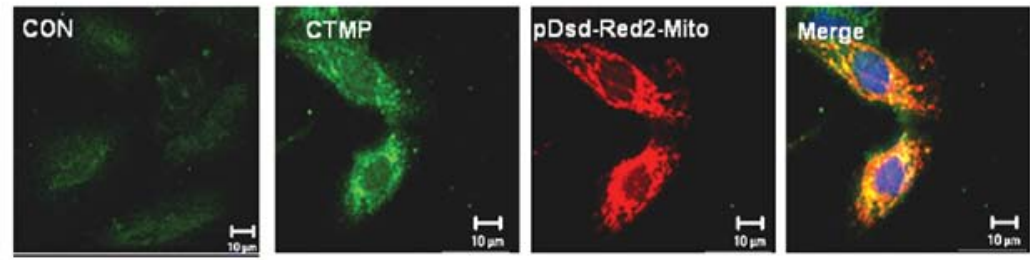

D
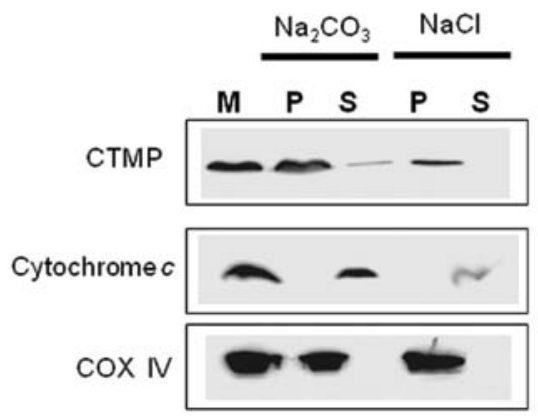

E
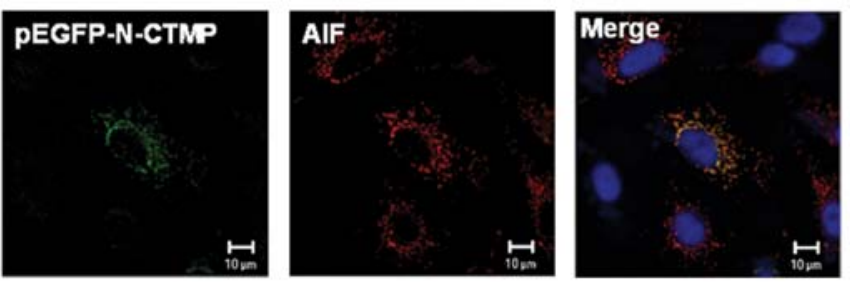

Figure 1. Localization of CTMP in mitochondria. (A) Cytoplasm and mitochondria fractions were isolated from A549 cells. Total lystates, the cytosolic fraction, and mitochondria were analyzed by Western blotting (COXIV-mitochondria marker and $\alpha$-tubulin-cytosolic marker). (B) A549 cells were transiently co-transfected with pEGFP-N1-CTMP and pDsRed-Mito for $24 \mathrm{~h}$, and cells were observed using confocal microscopy. (C) A549 cells stably expressing pDsRed-Mito were infected with lentivirus-CTMP for $24 \mathrm{~h}$ and immunostained with anti-CTMP antibody. (D) The mitochondria fraction from A549 cells was isolated and treated with the indicated reagents. Samples were separated into supernatant (S) and precipitate (P) fractions, and then analyzed by immunoblotting with the indicated antibodies (cytochrome $c$, mitochondria membrane space protein; and COXIV, mitochondria membrane protein). (E) Immunofluorescence assay of apoptosis-inducing factor (AIF). A549 cells were grown on glass coverslips and transiently transfected with pEGFP-N1-CTMP. The cells were then stained with anti-AIF antibody (scale bar, $10 \mu \mathrm{m})$. 
A

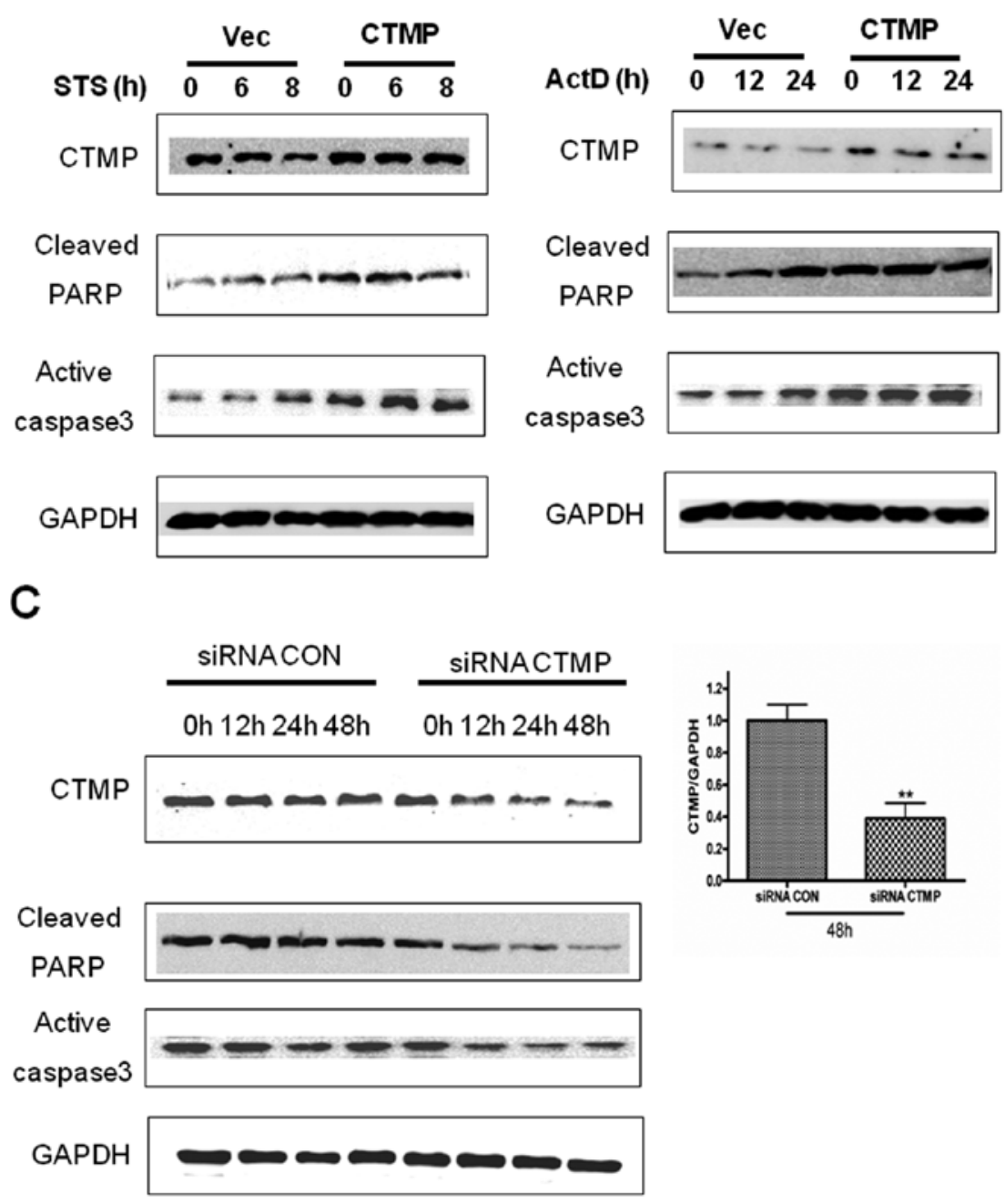

D

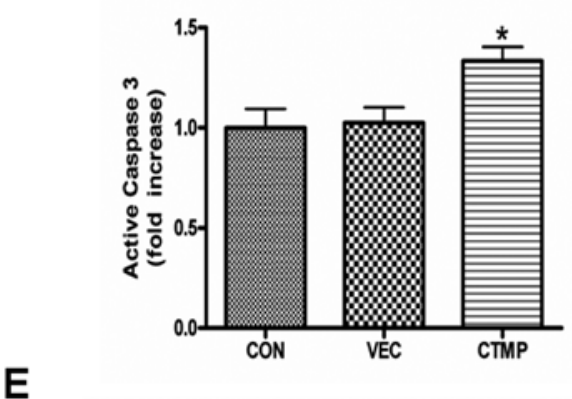

$\mathbf{F}$
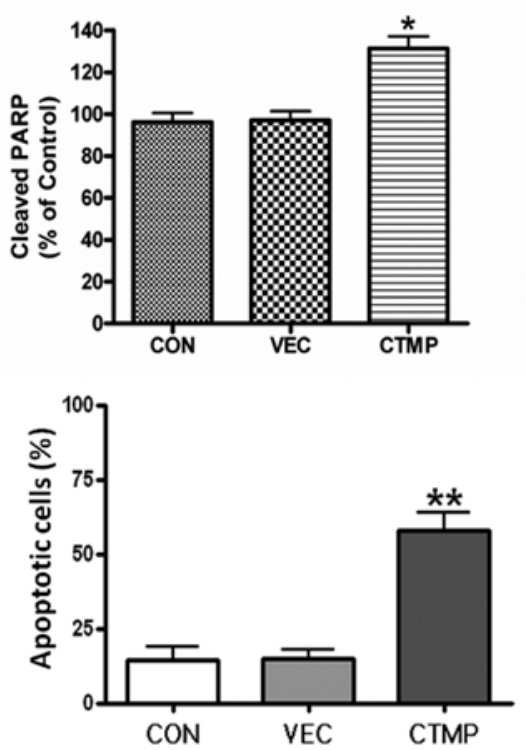

Figure 2. CTMP induces apoptosis in A549 cells. (A) A549 cells were infected with lentivirus-CTMP for $24 \mathrm{~h}$ and treated with $1 \mu \mathrm{M}$ staurosporine for the indicated times $(6$ and $8 \mathrm{~h})$. (B) A549 cells were infected with lentivirus-CTMP for $24 \mathrm{~h}$ and treated with $0.2 \mu \mathrm{M}$ actinomycin D for the indicated times $(12$ and $24 \mathrm{~h})$. (C) Western blot analysis was performed to measure CTMP expression in A549 cells after siRNA transfection for the indicated times. Right panel, densitometric analysis of CTMP expression in A549 cells after siRNA transfection for $48 \mathrm{~h}$. Each bar represents the mean \pm SE ( $=3$ ), ${ }^{*} \mathrm{p}<0.05$ and ${ }^{* *} \mathrm{p}<0.01$ compared to the corresponding control values and Western blot analysis measuring the levels of cleaved PARP and active caspase-3 was performed. (D) ELISA assay of active caspase-3. (E) ELISA assay of cleaved PARP. CON, untreated A549 cells; VEC, A549 cells infected with the lentivirus expression vector (VEC); CTMP, A549 cells infected with lentivirus-CTMP (CTMP). (F) Quantitative analysis of TUNEL-positive cells. Each bar represents the mean \pm SE $(n=3),{ }^{*} p<0.05$ and ${ }^{* *} \mathrm{p}<0.01$ compared to the corresponding control values.

normal cristae structures $(11,12)$. To detect the mitochondrial morphologic changes after CTMP overexpression, TEM and confocal laser scanning microscopy (CLSM) were performed. TEM analysis clearly showed the disorganization of mitochondria cristae in CTMP-infected cells (Fig. 3C). Overexpression of CTMP in A549 cells caused mitochondria fragmentation as observed by CLSM (Fig. 3D). Taken together, our results clearly demonstrated that CTMP caused mitochondrial morphologic changes.

CTMP decreases mitochondria membrane potential and release of cytochrome $c$ from mitochondria. It has been reported that OPA1 downregulation is associated with mitochondria membrane potential and apoptosis (13). Therefore, we measured changes of mitochondria membrane potential. Our result showed that infection with lentivirus-CTMP significantly reduced mitochondrial membrane potential compared to control cells (Fig. 4A). Recent studies have reported that OPA1 downregulation altered cristae structures by disrupting the formation of OPA1 oligomers at cristae junctions, thereby promoting the release of additional pools of cytochrome $c$ into the cytosol (14). We clearly observed that CTMP overexpression resulted in the release of cytochrome $c$ from mitochondria into the cytosol (Fig. 4B). These findings were further reconfirmed by densitometric analysis and ELISA (Fig. 4C-E). Immunofluorescence analysis clearly showed that lentivirus-CTMP increased the expression of cytochrome $c$ (Fig. 4F).

CTMP suppresses heat-shock protein (Hsp) expression. Hsp has been reported to regulate apoptosis and cell death (15). Moreover, 
A

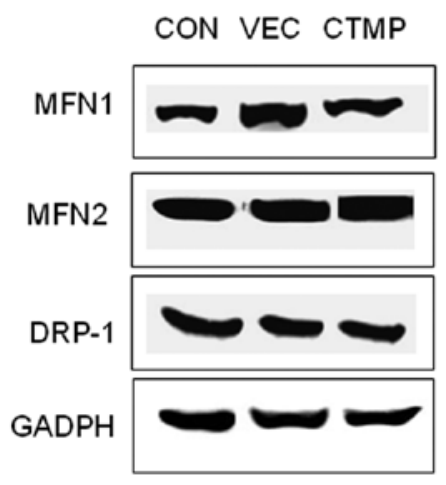

B

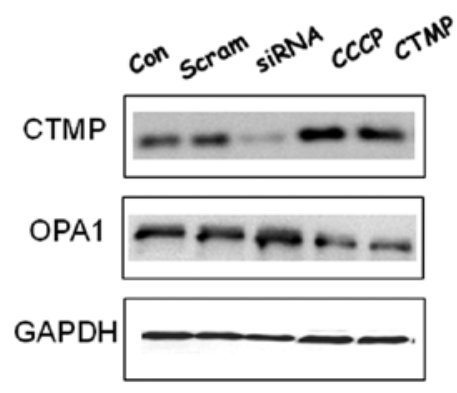

C
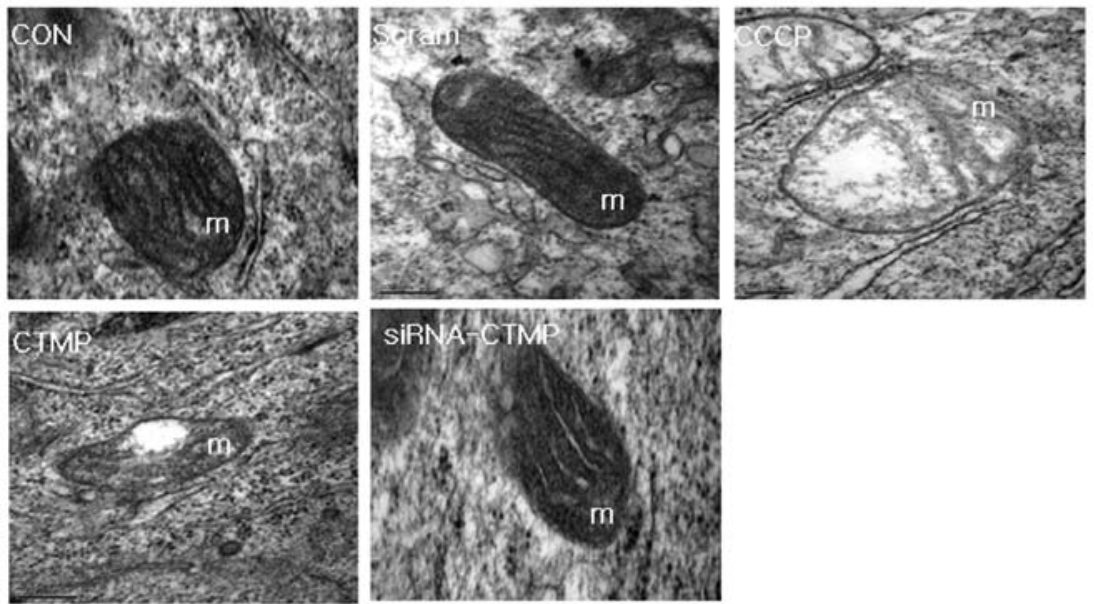

D
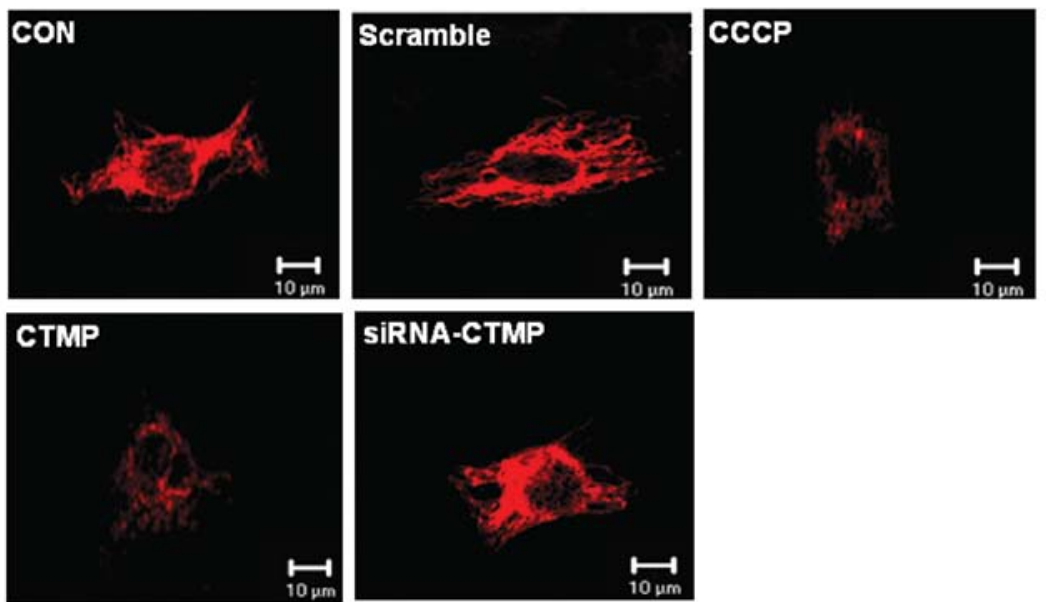

Figure 3. CTMP inhibits OPA1 expression and changes mitochondria morphology. (A) Western blot analysis of MFN1, MFN2, and DRP-1. A549 cells were infected with lentivirus-VEC/CTMP and cultured for $48 \mathrm{~h}$ before analysis. (B) A549 cells were infected with lentivirus-CTMP, treated with $0.2 \mu \mathrm{M}$ CCCP (carbonyl cyanide 3-chlorophenylhydrazone), and transfected with siRNA specific for CTMP. (C) The mitochondrial ultrastructure was analyzed by TEM. (D) A549 cells stably expressing mitochondrion-targeted pDsRed-Mito were infected with lentivirus expression vector and lentivirus-CTMP for $48 \mathrm{~h}$ and observed with confocal microscopy (scale bar, $10 \mathrm{~nm}$ ).

a recent study revealed a possible interaction of CTMP with Hsp (16). Hsp is known to influence apoptosis through direct physical interaction with key components of the apoptotic machinery (17). Therefore, we investigated the changes in the expression of Hsp27 and Apaf-1. Our results showed that CTMP clearly decreased Hsp 27 protein level and increased Apaf-1 expression as determined by Western blotting, immunofluorescence and FACS analyses (Fig. 5). 
A
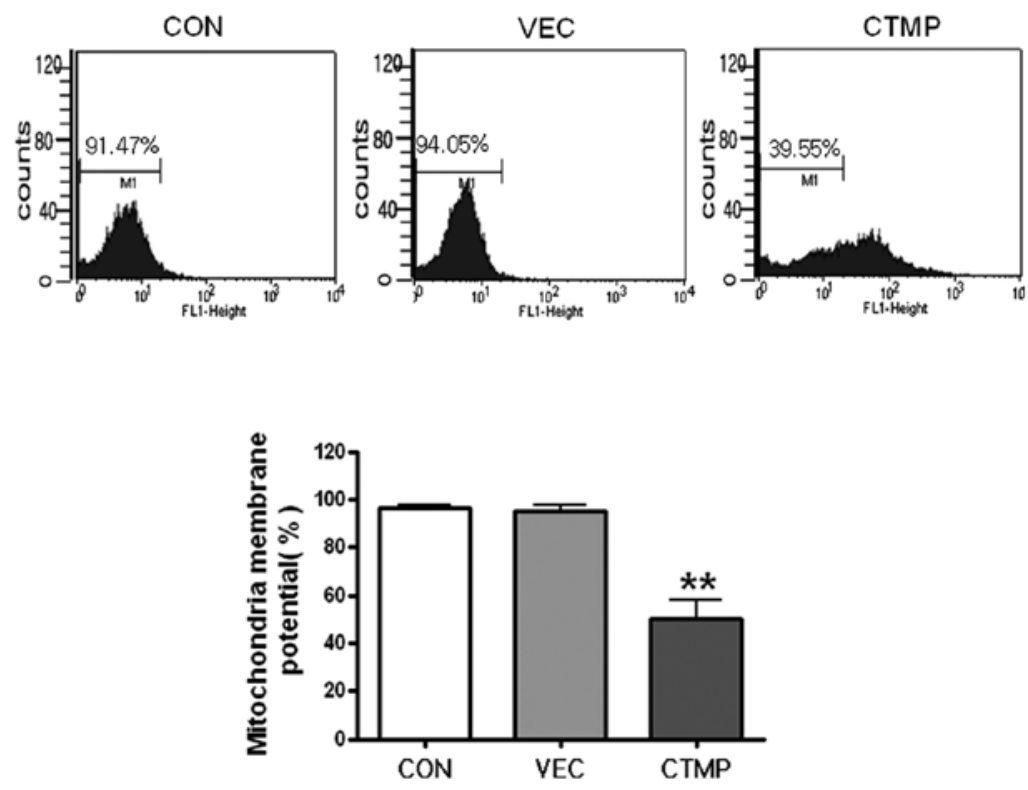

B
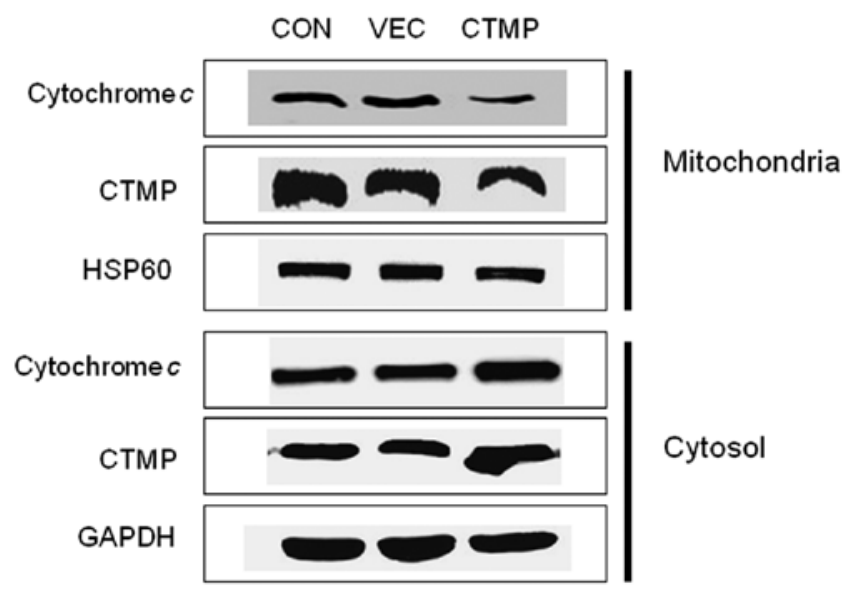

C
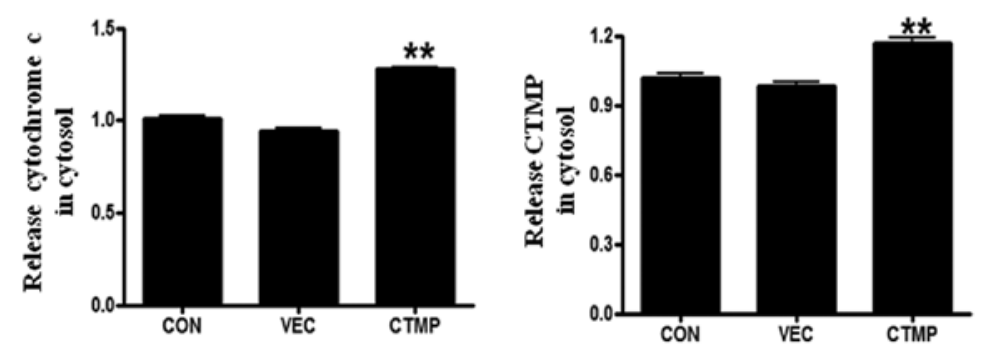

Figure 4. CTMP reduces mitochondria membrane potential that leads to cytochrome $c$ release. (A) CTMP-infected cells were stained using $5 \mu \mathrm{g} / \mathrm{ml} \mathrm{JC}-1$ and analyzed by flow cytometry. (B) Western blot analysis of CTMP and cytochrome $c$ in mitochondria. (C and D) The bands of interest were further analyzed by densitometry. Each bar represents the mean $\pm \mathrm{SE}(\mathrm{n}=3),{ }^{*} \mathrm{p}<0.05$ and ${ }^{* *} \mathrm{p}<0.01$ compared to the corresponding control values.

\section{Discussion}

The mitochondria are organelles important for cell growth and energy production. In addition, mitochondria participate in signaling events that influence numerous processes such as cell proliferation and death $(10,18)$. In fact, mitochondria have emerged as an effective target for anti-cancer therapy (19).

Akt signaling has been implicated in cancer progression due to its ability to regulate cellular proliferation and inhibit apoptosis (4). Akt regulates apoptosis, and directly 


\section{$\mathbf{E}$}

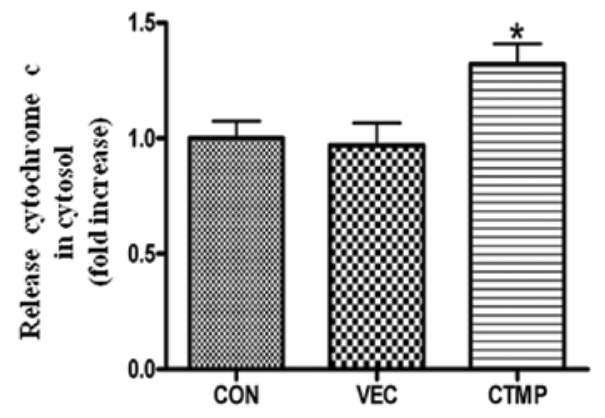

$\mathbf{F}$
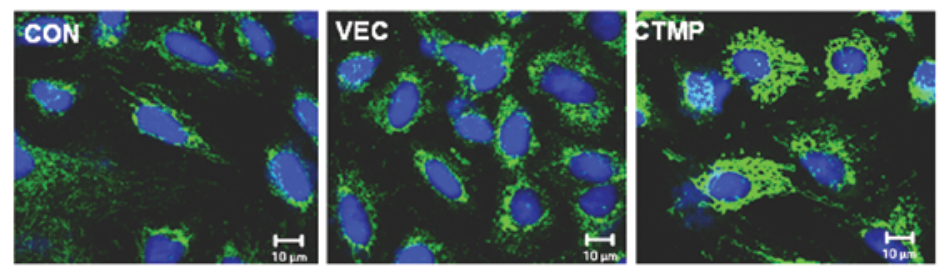

Figure 4. Continued. (E) ELISA to measure cytochrome $c$ in the cytosol. (F) Immunofluorescence assay of cytochrome $c$.

A

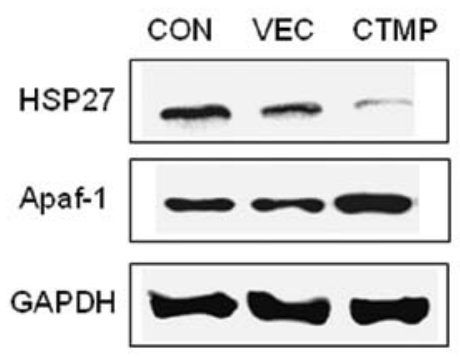

B
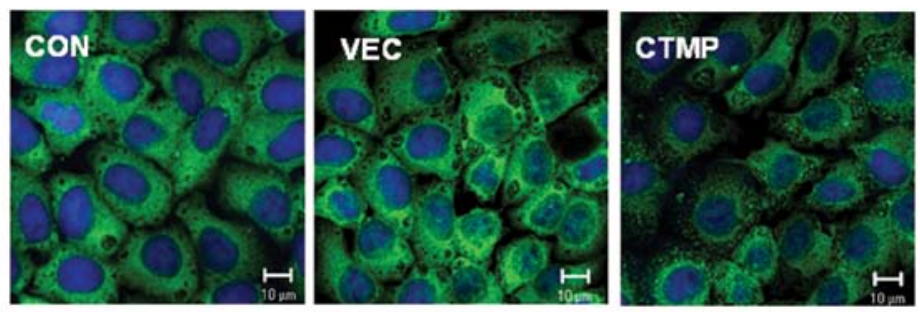

C
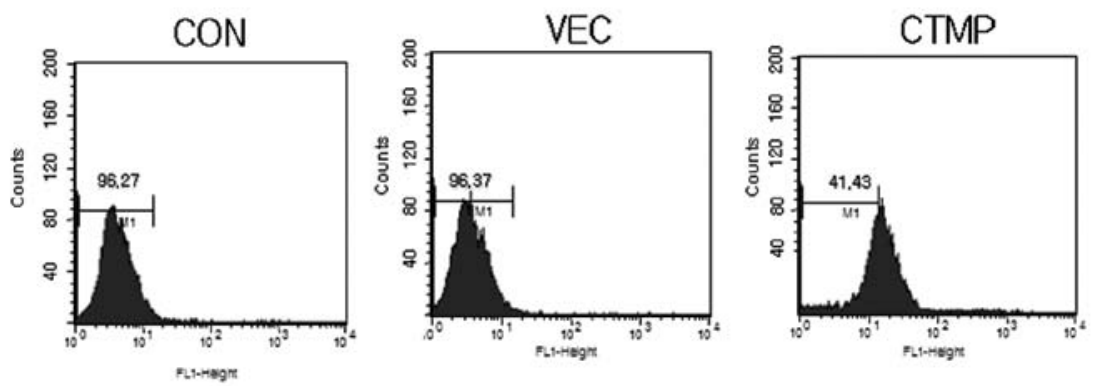

Figure 5. CTMP inhibits heat-shock proteins. A549 cells were infected with lentivirus-VEC/CTMP and cultured for 48 h. (A) Western blot analysis of Hsp27 and Apaf-1. (B) Immunofluorescence assay of Hsp27. (C) FACS analysis of Apaf-1 in CTMP-infected cells. 
phosphorylates and interacts with key factors involved in mitochondria-mediated apoptosis signaling (20). Moreover, Akt signaling has been found to be involved in the survival and proliferation of a variety of tumor cells (21). Akt activity is regulated by dual phosphorylation of regulatory residues, Thr308 in the activation loop of the catalytic domain and Ser473 in the regulatory domain (22). CTMP negatively regulates Akt activity by inhibiting Ser473 and Thr308 phosphorylation (7). Previously, we have demonstrated that CTMP suppresses lung cancer progression by inhibiting Akt activity and inducing apoptosis in a murine lung cancer model (23). A recent study demonstrated that CTMP interacts with LETM1, another protein involved in mitochondrial dynamics and apoptosis. Therefore, in this study we investigated the effect of CTMP on mitochondria function and apoptosis in lung cancer cells. It has been reported that the function of CTMP in mitochondria is tightly linked to its submitochondrial distribution (15). Taken together, our results clearly demonstrated that overexpression of CTMP induced apoptosis in lung adenocarcinoma cells (Fig. 2). During apoptosis, the mitochondrial network fragments, resulting in smaller and more numerous mitochondria (3). It has been reported that increased fission, decreased fusion, or both cause this at a stage of apoptosis prior to caspase activation and cytochrome $c$ release. Recent work showed that proteins involved in mitochondrial fission and fusion also actively participate in apoptosis (4). This is why we investigated the effects of CTMP on mitochondria fission and fusion. We analyzed the expression of mitochondria dynamics-related protein such as MFN1, MFN2, OPA1 (mitochondria fusion), and DRP-1 (mitochondria fission). We observed that CTMP did not alter the expression of mitochondria fusion proteins such as MFN1, 2, and mitochondria fission protein such as DRP-1 (Fig. 3A). Rather, CTMP decreased OPA1 expression (Fig. 3B). Downregulation or knockout of mammalian OPA1 is known to promote the disorganization of inner membrane cristae, thereby inducing overall mitochondrial fragmentation and inhibiting mitochondrial fusion $(24,25)$. Our results closely correlate with these previous report because we clearly observed the disorganization of mitochondrial cristae (Fig. 3C) and fragmented mitochondria in cells overexpressing CTMP (Fig. 3D). These results suggested that CTMP altered mitochondria morphology by inhibiting OPA1 expression.

A hallmark of the mitochondria apoptotic pathway is the release of cytochrome $c$ followed by changes in mitochondria morphology and permeability (26). Recently, it was hypothesized that mitochondrial cristae reorganization is required for the redistribution of cytochrome $c$ normally sequestered from the intermembrane space within the cristae folds, thereby allowing the release of cytochrome $c$ during apoptosis (27). We also demonstrated that CTMP overexpression induced cytochrome $c$ release from mitochondria into the cytosol and reduced membrane potential (Fig. 4). These results indicated that CTMP may play an important role in mitochondriamediated apoptosis.

Cell death signaling induces cytochrome $c$ release from the mitochondria, which then binds to apoptosis protease activating factor-1 (Apaf-1) which induces oligomerization and apoptosome formation (17). Hsp27 may inhibit apoptosis by directly binding to cytosolic cytochrome $c$ and sequestering it from Apaf-1 (28). In fact, we showed that overexpression of CTMP inhibited Hsp27 and increased Apaf-1 expression (Fig. 5).

Taken together, our results demonstrated that CTMP overexpression induced mitochondria-mediated apoptosis through downregulation of OPA1 and inhibition of Hsp 27. Further studies are required to elucidate the detailed role of CTMP as a promising pro-apoptotic factor that may critically influence lung cancer cell death.

\section{Acknowledgements}

This work was supported partially by a research grant (NRF2010-0000784, 2011-0019175 and NRF-2011-0000380) from the National Research Foundation of the Ministry of Education, Science and Technology in South Korea. This study was also partially supported by the Research Institute for Veterinary Science of Seoul National University.

\section{References}

1. Densmore CL: Advances in noninvasive pulmonary gene therapy. Curr Drug Deliv 3: 55-63, 2006.

2. Chan DC: Mitochondria: dynamic organelles in disease, aging, and development. Cell 125: 1241-1252, 2006.

3. Karbowski M and Youle RJ: Dynamics of mitochondrial morphology in healthy cells and during apoptosis. Cell Death Differ 10: 870-880, 2003 .

4. Lawlor MA and Alessi DR: PKB/Akt: a key mediator of cell proliferation, survival and insulin responses? J Cell Sci 114: 2903-2910, 2001.

5. Sasaki K, Sato M and Umezawa Y: Fluorescent indicators for $\mathrm{Akt} /$ protein kinase $\mathrm{B}$ and dynamics of Akt activity visualized in living cells. J Biol Chem 278: 30945-30951, 2003.

6. Franke TF, Kaplan DR and Cantley LC: PI3K: downstream AKTion blocks apoptosis. Cell 88: 435-437, 1997.

7. Maira SM, Galetic I, Brazil DP, et al: Carboxyl-terminal modulator protein (CTMP), a negative regulator of PKB/Akt and v-Akt at the plasma membrane. Science 294: 374-380, 2001.

8. Dryer SE, Chae KS, Martin-Caraballo M and Anderson M: Akt activation is necessary for growth factor-induced trafficking of functional K-Ca channels in developing parasympathetic neurons. J Neurophysiol 93: 1174-1182, 2005.

9. Hwang SK, Kwon JT, Park SJ, et al: Lentivirus-mediated carboxylterminal modulator protein gene transfection via aerosol in lungs of K-ras null mice. Gene Ther 14: 1721-1730, 2007.

10. Suen DF, Norris KL and Youle RJ: Mitochondrial dynamics and apoptosis. Genes Dev 22: 1577-1590, 2008.

11. Frezza C, Cipolat S, Martins de Brito O, et al: OPA1 controls apoptotic cristae remodeling independently from mitochondrial fusion. Cell 126: 177-189, 2006.

12. Scorrano L and Pellegrini L: A cut short to death: Parl and Opa1 in the regulation of mitochondrial morphology and apoptosis. Cell Death Differ 14: 1275-1284, 2007.

13. Pellegrini L and Scorrano L: A cut short to death: Parl and Opa1 in the regulation of mitochondrial morphology and apoptosis. Cell Death Differ 14: 1275-1284, 2007.

14. Arnoult D, Grodet A, Lee YJ, Estaquier J and Blackstone C: Release of OPA1 during apoptosis participates in the rapid and complete release of cytochrome $\mathrm{c}$ and subsequent mitochondrial fragmentation. J Biol Chem 280: 35742-35750, 2005.

15. Piao L, Li Y, Yang KJ, et al: Heat shock protein 70-mediated sensitization of cells to apoptosis by carboxyl-terminal modulator protein. BMC Cell Biol 10: 53, 2009.

16. Beere HM: Stressed to death: regulation of apoptotic signaling pathways by the heat shock proteins. Sci STKE 2001: re1, 2001.

17. Takayama S, Reed JC and Homma S: Heat-shock proteins as regulators of apoptosis. Oncogene 22: 9041-9047, 2003.

18. Desagher S and Martinou JC: Mitochondria as the central control point of apoptosis. Trends Cell Biol 10: 369-377, 2000.

19. Hogg PJ and Don AS: Mitochondria as cancer drug targets. Trends Mol Med 10: 372-378, 2004.

20. Hemmings BA, Parcellier A, Tintignac LA and Zhuravleva E: PKB and the mitochondria: AKTing on apoptosis. Cell Signal 20: 21-30, 2008. 
21. Vivanco I and Sawyers CL: The phosphatidylinositol 3-kinase AKT pathway in human cancer. Nat Rev Cancer 2: 489-501, 2002.

22. Hennessy BT, Smith DL, Ram PT, Lu Y and Mills GB: Exploiting the PI3K/AKT pathway for cancer drug discovery. Nat Rev Drug Discov 4: 988-1004, 2005.

23. Hwang SK, Lim HT, Minai-Tehrani A, et al: Repeated aerosol delivery of carboxyl-terminal modulator protein suppresses tumor in the lungs of K-rasLA1 mice. Am J Respir Crit Care Med 179: 1131-1140, 2009.

24. Lee YJ, Jeong SY, Karbowski M, Smith CL and Youle RJ: Roles of the mammalian mitochondrial fission and fusion mediators Fis1, Drp1, and Opa1 in apoptosis. Mol Biol Cell 15: 5001-5011, 2004.
25. Merkwirth C, Dargazanli S, Tatsuta T, et al: Prohibitins control cell proliferation and apoptosis by regulating OPA1-dependent cristae morphogenesis in mitochondria. Genes Dev 22: 476-488, 2008.

26. Wang XD: The expanding role of mitochondria in apoptosis. Gene Dev 15: 2922-2933, 2001.

27. Olichon A, Baricault L, Gas N, et al: Loss of OPA1 perturbates the mitochondrial inner membrane structure and integrity, leading to cytochrome c release and apoptosis. J Biol Chem 278: 7743-7746, 2003.

28. Bruey JM, Ducasse C, Bonniaud P, et al: Hsp27 negatively regulates cell death by interacting with cytochrome c. Nat Cell Biol 2: 645-652, 2000. 